Kenneth R. Kellum, Department of Mathematics and Computer Science, San José State University, San José, California 95192-0103,

e-mail: kellum@sjsumcs.sjsu.edu

\title{
COMPOSITIONS OF DARBOUX-LIKE FUNCTIONS
}

\begin{abstract}
An example is given of a real connectivity function which is not the composition of any finite collection of almost continuous functions. We also investigate conditions under which the composition of two real Darboux functions can be continuous.
\end{abstract}

In [6] I asked if every Darboux function from $\mathbb{R}$ to $\mathbb{R}$ is the composition of two almost continuous functions. In the present note it is shown that the answer is "no". In fact, there exits a connectivity function which cannot be written as the composition of finitely many almost continuous functions. This example is not particularly difficult. I think we have overlooked this example until now because no one expected it to exist. The composition of almost continuous functions can be very nasty. Our function which is not such a composition is as well behaved as a Darboux function can be and not be almost continuous.

Natkaniec [7] has shown that if $f$ is Darboux and nasty-that is, $f^{-1}(x)$ is c-dense for every $x$, then $f$ is the composition of two almost continuous functions. To look at the other extreme, we need a Darboux function which is not almost continuous but as nice as possible. Since a Darboux function of Baire class 1 is almost continuous [1], the function we want must be totally discontinuous on some perfect set. Jones and Thomas [5] give an example of a function which is connectivity, continuous on the complement of the Cantor set, but not almost continuous. Our example is a simple modification of the Jones and Thomas example.

A function $f: \mathbb{R} \rightarrow \mathbb{R}$ is almost continuous if, given a closed set $K \subset \mathbb{R}^{2}$ such that $\operatorname{graph}(f) \cap K=\emptyset$, there exists a continuous function $g: \mathbb{R} \rightarrow \mathbb{R}$ such that $\operatorname{graph}(g) \cap K=\emptyset . f$ is Darboux if $f(C)$ is connected whenever $C$ is connected. $f$ is a connectivity function if graph $\left(\left.f\right|_{C}\right)$ is connected whenever $C$

Key Words: almost continuous function, Darboux function, connectivity function

Mathematical Reviews subject classification: Primary 26A15; Secondary 54C30

Received by the editors July 8, 1997 
is connected. In this setting, an almost continuous function is a connectivity function and a connectivity function is Darboux. The reader interested in learning about these classes of functions should see the excellent survey articles [4], [7] and [2].

We begin with two technical lemmas. The letter $I$ denotes the closed unit interval $[0,1]$.

Lemma 1. Suppose $f: I \rightarrow I, U \subset I$ is an open interval, $f^{-1}(U)$ is open and not connected, $f$ is continuous on each component of $f^{-1}(U)$ and $f$ is either increasing on every component of $f^{-1}(U)$ or is decreasing on all of them. Then $f$ cannot be almost continuous.

Proof. ${ }^{1}$ If $f$ is not Darboux, we are done; so we may assume that it is.

Assume $f$ is increasing on components of $f^{-1}(U)$. Choose a closed interval $[p, q] \subset U$. Pick two components $[a, b]$ and $[c, d]$ of $f^{-1}([p, q])$. The closed set $K_{1}=(I \times[p, q]) \cup(\{a, c\} \times[q, 1]) \cup(\{b, d\} \times[0, p])$ intersects the graph of every continuous function from $I$ into itself.

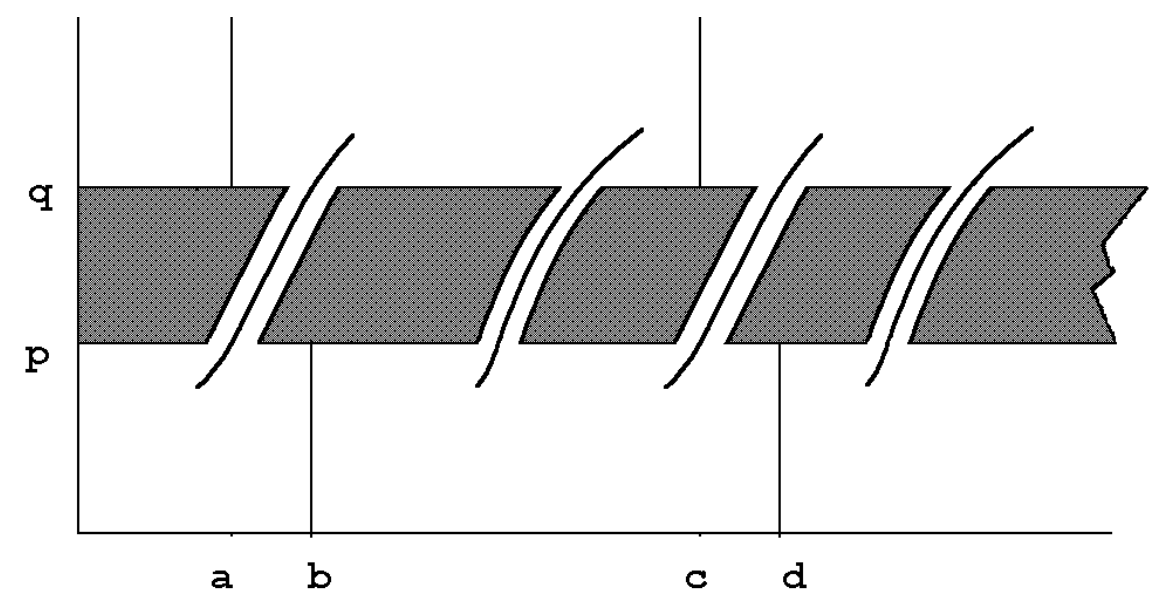

Figure 1: The closed set $\mathrm{K}$

For each component $(u, v)$ of $f^{-1}(U)$, we will remove a "tube" from $K_{1}$. (See Figure 1.) Since $f(u)$ and $f(v)$ cannot be in $U$, and $f([u, v])$ is connected, it follows that $f((u, v))=U$. Let $s$ and $t$ denote the unique points in $(u, v)$ such that $f(s)=p$ and $f(t)=q$. Choose $\epsilon>0$ so that $u<s-\epsilon<s+\epsilon<t-\epsilon<$

\footnotetext{
${ }^{1}$ I thank the referee and Professors Jack Brown and Krzysztof Ciesielski for pointing out errors in earlier version of this proof.
} 
$t+\epsilon<v$. Let $V_{u, v}$ be the union of all open line segments $(x-\epsilon, x+\epsilon) \times\{f(x)\}$ centered at points $(x, f(x))$, where $s \leq x \leq t$. Finally, let $K=K_{1} \backslash \bigcup V_{u, v}$, where the union is taken over all components of $f^{-1}(U) . K$ is a closed set, $K \cap \operatorname{graph}(f)=\emptyset$ and $K$ intersects the graph of every continuous function from $I$ into itself. Thus, $f$ is not almost continuous.

Lemma 2. Suppose $f: I \rightarrow I$ and that there exist nondegenerate closed intervals, $[a, b],[c, d],[p, q]$ and $[u, v]$ such that $f^{-1}([p, q])=[a, b], f^{-1}([u, v])=$ $[c, d],[a, b] \cap[c, d]=\emptyset, f$ is continuous $[a, b] \cup[c, d], f$ is increasing on $[a, b]$ and is decreasing on $[c, d]$. Then $f$ is not almost continuous.

Proof. Choose $x$ and $y$ such that $a<x<b$ and $c<y<d$. Then $(I \times$ $[p, q]) \cup(\{x\} \times[q, 1]) \cup(\{x\} \times[0, p]) \cup(I \times[u, v]) \cup(\{y\} \times[v, 1]) \cup(\{y\} \times[0, u])$ is a closed set which intersects the graph of every continuous function from $I$ into $I$. We can now remove a tube from $I \times[p, q]$ above $[a, b]$ and a tube from $I \times[u, v]$ above $[c, d]$ and obtain a closed set with misses graph $(f)$ but which intersects the graph of every continuous function from $I$ into $I$.

Let $\mathcal{C}$ denote the Cantor middle-thirds set. By a complementary interval we mean the closure of a component of $I \backslash \mathcal{C}$. We define a function $j: I \rightarrow I$ as follows. For each complementary interval $[a, b]$, choose $\left.j\right|_{[a, b]}$ to be continuous and increasing with $j(a)=0$ and $j(b)=1$. Let $\mathcal{C}^{\circ}$ denote the points of $\mathcal{C}$ which are not endpoints of complementary intervals. If $x \in \mathcal{C}^{\circ}$, let $j(x)=0$. It follows from Lemma 1 that $j$ is not almost continuous. Note that $j$ is a connectivity function.

Example 1. : The function $j$ is not the composition of any finite collection of almost continuous functions from I to $I$.

Proof. Assume that it is. Let $j=f_{n} \circ \cdots \circ f_{1}$, where $f_{i}$ is almost continuous.

Consider some complementary interval, $[a, b]$. Since $j$ is one-to-one on $[a, b]$, $f_{1}$ must also be one-to-one there. Since a one-to-one Darboux function on an interval is continuous, it follows that $f_{1}$ is monotone increasing or decreasing on $[a, b]$. Thus, $f_{1}([a, b])$ is a closed interval, and, by the same reasoning, $f_{2}$ is one-to-one and continuous on $f_{1}([a, b]), f_{3}$ is one-to-one and continuous on $f_{2} \circ f_{1}([a, b])$, and so on.

Let $\mathcal{P}_{0}$ denote the set of complementary intervals. For $i=1, \ldots, n$, let $\mathcal{P}_{i}=\left\{f_{i}(P): P \in \mathcal{P}_{i-1}\right\}$. Each member of $\mathcal{P}_{i-1}$ is mapped homeomorphically by $f_{i}$ onto a member of $\mathcal{P}_{i}$.

Suppose $P, Q \in \mathcal{P}_{1}$. Then $f_{n} \circ \cdots \circ f_{2}$ must map both $P$ and $Q$ homeomorphically onto $I$. The endpoints of these intervals, and only the endpoints, are mapped to 0 and 1 . Thus $P \cap Q$ is either empty or is a common endpoint. Similarly, for each $i$, the interiors of $\mathcal{P}_{i}$ are pairwise disjoint. 
Suppose $x \in \mathcal{C}^{\circ}$. Since $j(x)=0$, and interiors of members of $\mathcal{P}_{i}$ map onto interiors of $\mathcal{P}_{i+1}, f_{1}(x)$ is not in the interior of any member of $\mathcal{P}_{1}, f_{2} \circ f_{1}(x)$ is not in the interior of any member of $\mathcal{P}_{2}$, and so on. Thus, for each $P \in \mathcal{P}_{i+1}$, $f_{i}^{-1}(\operatorname{int}(P))$ is open, in fact, is the union of interiors of elements of $\mathcal{P}_{i}$.

Now, we will prove by induction that for each $i$ with $0<i \leq n$, no two members of $\mathcal{P}_{i-1}$ are mapped by $f_{i}$ onto the same member of $\mathcal{P}_{i}$ and that $f_{i}$ is either increasing on every member of $\mathcal{P}_{i}$ or is decreasing on all of them.

First, if $f_{1}([c, d])=f_{1}([a, b])$, since $f_{n} \circ \cdots \circ f_{1}(d)=f_{n} \circ \cdots \circ f_{1}(b)=1$, we must have $f_{1}(b)=f_{1}(d)$. Thus, if two or more elements of $\mathcal{P}_{0}$ map to the same element of $\mathcal{P}_{1}, f_{1}$ is increasing on all or is decreasing on all of these. By Lemma 1 , this is impossible. Now, by Lemma $2, f_{1}$ cannot be increasing on one element of $\mathcal{P}_{0}$ and decreasing on another.

Assume now that we have the desired conditions for all $i$ with $0<i \leq k-1$. Then $f_{k-1} \circ \cdots \circ f_{1}$ increases on all members of $\mathcal{P}_{0}$ or decreases on all of them. If $f_{k}([c, d])=f_{k}([a, b])$, we can reason as above to conclude that $f_{k}(c)=f_{k}(a)$. Lemma 1 then rules this possibility out. Apply Lemma 2 again to complete the induction.

By the above argument, $f_{i}$ induces a one-to-one correspondence between $\mathcal{P}_{i}$ and $\mathcal{P}_{i+1}$. Since $\mathcal{P}_{n}$ has $[0,1]$ as its only element, we have a contradiction.

Remark. Another way we could obtain a contradiction in the proof of Example 1 is as follows. First, choose $n$ to be the smallest integer such that $j$ is the composition of $n$ almost continuous functions. Since the composition of a continuous function and an almost continuous function is almost continuous, none of $f_{1}, \ldots, f_{n}$ could be continuous. Once we have that $f_{1}$ is increasing (decreasing) on each complementary interval, we can use an argument similar to the lemmas to show that $f_{1}$ is increasing (decreasing) on $I \backslash \mathcal{C}$. The next step is a little delicate, but, using the fact that $f_{1}$ sends points of $\mathcal{C}^{\circ}$ only to endpoints, we can then argue that $f_{1}$ is continuous.

In the proof of Example 1, we made repeated use of the fact that if the composition of two real Darboux functions is strictly monotone, then each factor is continuous and strictly monotone. It is natural to ask: If $g \circ f$ is continuous, where $g$ and $f$ are Darboux, then what can be said about $g$ and $f ?$

Example 2. There exist $f$ Darboux and discontinuous and $g$ continuous such that $g \circ f$ is continuous.

Define $f: I \rightarrow I$ as follows: Let $f\left(\mathcal{C}^{\circ}\right)=[0,1 / 2]$ so that for each $y \in$ $[0,1 / 2], f^{-1}(y)$ is c-dense in $\mathcal{C}$. For each complementary interval $[a, b]$, define $[a, b]$ to be continuous so that $f(a)=f(b)=1 / 2$ and $f([a, b])=[1 / 2,1]$. 
Define $g$ by $g(x)=0$ for $0 \leq x \leq 1 / 2$ and $g(x)=x-1 / 2$ otherwise.

We will now show that all such examples will be similar to the above, in that the discontinuities of one factor are crushed by intervals of constancy of the other.

By the cluster set of $f$ at $a$, denoted by $C(f, a)$, we mean the set of all $y$ such that $(a, y)$ is a limit point of the set $\{(x, f(x)): x \neq a\}$. The cluster sets of a Darboux function are connected.

Theorem 1. Suppose $f$ and $g$ are Darboux functions from $\mathbb{R}$ into itself and that $g \circ f$ is continuous at $a$. If $f$ is discontinuous at $a$, then $g$ is constant on $C(f, a)$.

Proof. Pick $y \in \operatorname{int}(C(f, a))$, where $y \neq f(a)$. Since $f$ is Darboux, there exists a sequence $\left\{x_{n}\right\}$ converging to $a$ such that $f\left(x_{n}\right)=y$. By hypothesis, $g \circ f\left(x_{n}\right)$ converges to $g \circ f(a)$. Since $g \circ f\left(x_{n}\right)=g(y)$, we must have $g(y)=g \circ$ $f(a)$. Since $g$ is constant on int $(C(f, a))$ and is Darboux, the result follows.

Theorem 2. Suppose $f$ and $g$ are Darboux functions from $\mathbb{R}$ into itself and that $g \circ f$ is continuous at $a$. If $g$ is discontinuous from the left (right) at $f(a)$, then $f$ has a local minimum (maximum) at $a$. Thus, if $g$ is discontinuous from both sides at $f(a)$, then $f$ is constant in a neighborhood of $a$.

Proof. Assume $g$ is discontinuous from the left at $f(a)$. If $f$ does not have a local minimum at $a$, there exists a sequence $\left\{x_{n}\right\}$ converging to $a$, where $f\left(x_{n}\right)<f(a)$. Because $f$ is Darboux, we may choose $\left\{x_{n}\right\}$ so that $\left\{f\left(x_{n}\right)\right\}$ converges to $f(a)$. Since $g$ is Darboux, there exists an increasing sequence $\left\{y_{n}\right\}$ converging to $f(a)$ where $g\left(y_{n}\right)=y$ for each $n$ and $y \neq g \circ f(a)$. Passing to a subsequence, we may assume that $y_{n}>f\left(x_{n}\right)$. Since $f$ is Darboux, there exists $z_{n}$ between $x_{n}$ and $a$ such that $f\left(z_{n}\right)=y_{n}$. But then, $g \circ f\left(z_{n}\right)=y$, contradicting the continuity of $g \circ f$ at $a$.

Theorem 3. Suppose $f$ and $g$ are Darboux functions from $\mathbb{R}$ to $\mathbb{R}$ where $f$ is onto. If $g \circ f$ is continuous, then $g$ is continuous.

Proof. Assume, on the contrary, that $g$ is discontinuous at $a$, from, say, the right. For each $x$ such that $f(x)=a, f$ has a maximum at $x$.

First, we claim that $f^{-1}((-\infty, a])$ is closed. For, if $x$ was a limit point of $f^{-1}((-\infty, a])$ and $f(x)>a$, then $f$ would be discontinuous at $x$ and $[a, f(x)] \subset$ $C(f, x)$. By Theorem $1, g$ would then be constant on $[a, f(x)]$, a contradiction.

Now, pick $z \in f^{-1}(a+1)$. At least one of $f^{-1}((-\infty, a]) \cap(-\infty, z]$ and $f^{-1}((-\infty, a]) \cap[z, \infty)$ is nonempty, so assume $f^{-1}((-\infty, a]) \cap(-\infty, z] \neq \emptyset$. Let $t=\max \left(f^{-1}((-\infty, a]) \cap(-\infty, z]\right)$. Then $f(t) \leq a$. If $f(t)<a$, we have 
a contradiction because $f$ is Darboux. If $f(t)=a$, we have a contradiction because $f$ must have a maximum at $t$.

The techniques of this paper do not seem to apply to the following question, due to Ceder [3], which remains open.

Question. Is every real Darboux function the composition of two (finitely many) connectivity functions?

\section{References}

[1] J. B. Brown, Almost continuous Darboux functions and Reed's pointwise convergence criteria, Fund. Math., 86 (1974), 1-7.

[2] A. M. Bruckner and J. G. Ceder, Darboux continuity, Jahresber. Deutsch. Math-Verein., 67 (1965), 93-117.

[3] J. G. Ceder, On composition with connected functions, Real Analysis Exchange 11 (1985-86), 380-390

[4] R. G. Gibson and T. Natkaniec, Darboux like functions, Real Analysis Exchange 22 (2), (1996-97), 1-42.

[5] F. B. Jones and E. S. Thomas, Jr., Connected $G_{\delta}$-graphs, Duke Math. J., 33 (1966), 341-345.

[6] K. R. Kellum, Iterates of almost continuous functions and Sarkovskii's Theorem, Real Analysis Exchange, 14, (1988-89), 420-423.

[7] T. Natkaniec, Almost continuity, Real Analysis Exchange, 17 (1991-92), $462-520$. 